\title{
Effect of Psychological Care Combined with Prozac on Patients with Post-Stroke Depression
}

\author{
Jimei $\mathbf{L i}^{*}$ \\ Department of Neurology, Affiliated hospital of Xuzhou Medical College, Xuzhou, Jiangsu 21003, China
}

\begin{abstract}
Objective: To observe the effects of psychological care combined with Prozac on patients with post-stroke depression. Methods: 60 patients with post-stroke depression were divided randomly into two groups: experimental group and control group. The treatment group was treated with psychological care combined with Prozac and the control group was treated with Prozac itself. The Hamailton Depression Rating Scale (HAMD) and Ability of Daily Life (ADL) before treatment and six weeks later were determined. Results: After treatment, the depression symptom of two groups were improved obviously $(p<0.01)$ and ADL was raised $(p<0.01)$. Compared with Prozac itself, psychological care combined with Prozac was much more effective $(p<0.05)$. Conclusions: The psychological care combined with Prozac has better effect on patients with post-stroke depression.
\end{abstract}

\section{KEYWORDS}

Post-Stroke depression

Psychological treatment

Prozac

\section{Introduction}

Post-Stroke Depression (PSD) is the most common complications of cerebral apoplexy, which affecting recovery of patients' neurological function, and reducing patient's quality of life. Positive psychological care and drug treatment of patients not only improve the depressive state of patients with cerebral apoplexy, but also improve ability of patients' daily living, these directly beneficial to improve patients' quality of life. Now report the results as follows.

\section{Materials and methods}

\subsection{Clinical data}

\subsubsection{Inclusion criteria}

(1) Patients with cerebral apoplexy for the first time, who met the diagnosis standards of cerebral apoplexy developed by the fourth national academic conference on cerebrovascular disease in 1995 [1]. (2) There was evidence of cerebral hemorrhage or cerebral ischemia in brain CT

\section{Copyright $\odot 2016$ Jimei Li}

doi: 10.18686/jn.v5i4.6

Received: September 19, 2016; Accepted: November 3, 2016; Published online: December 29, 2016

This is an open-access article distributed under the terms of the Creative Commons Attribution Unported License (http://creativecommons.org/ licenses/by-nc/4.0/), which permits unrestricted use, distribution, and reproduction in any medium, provided the original work is properly cited.

${ }^{\star}$ Corresponding author: Department of Neurology, Affiliated hospital of Xuzhou Medical College, Xuzhou, Jiangsu 221003, China. E-mail: jimei_li@sina.com or MRI. (3) Patients without the history of mental illness and family history, epilepsy, brain trauma and other serious body disease. (4) Patients with clear consciousness and certain expression ability can talk smoothly, patients with aphasia or couldn't understand conversations were excluded. (5) The age $<75$ years old. (6) The self-evaluation depression scale (SDS) $\geq 30$ points and HAMD score $\geq 8$ points when admitted. (7) Patients that fulfil the standards of Chinese classification and diagnostic criteria of mental disorders, version 2 (CCMD-2-R).

\subsubsection{General information}

60 patients treated in our department of neurology which hospitalized since June 2014 to June 2015. 28 cases in the experimental group, included 14 males and 14 females, with average age of $(55.6 \pm 7.2)$ years old and 32 cases in the control group, included 18 males and 14 females, with average age of $(56.3 \pm 5.9)$ years old. There were no significant difference in age, gender, ADL and HAMD scores and other aspects between two groups $(p<0.05)$, therefore the data was comparable.

\subsection{Methods}

On the basis of conventional cerebrovascular drug treatment and rehabilitation therapy, two groups of patients were on Prozac treatment, which began with oral dose of $10 \mathrm{mg} / \mathrm{d}$, and gradually increases to the therapeutic dose of 20 to $40 \mathrm{mg} / \mathrm{d}$ in a week. The dose of experimental group was $(30.39 \pm 4.34) \mathrm{mg} / \mathrm{d}$, the control group was $(29.0 \pm$ 
3.78) $\mathrm{mg} / \mathrm{d}$. There was no significant difference between two groups $(p<0.05)$. Psychological care included: (1) Listen to the psychological feelings of patients carefully. (2) Accurate empathy; (3) Confrontation; When patients' words were not matched by deeds, point out its contradictions and conflicts. Encourage patients to leave their psychological defense, face themselves truly, promoting the unity of the reality and behavior; (4) Correctly understand the disease and build confidence to overcoming disease, by encourage patients to lift their spirits, involve themselves into the rehabilitation exercise, and establish a positive health belief; (5) Meet the needs of patients, tell them that they will recover from dysfunction after stroke through rehabilitation training. At the same time, explain to the patient the characteristic, prognosis and treatment methods and other related knowledge as well as the basic knowledge of rehabilitation function training of cerebrovascular disease, at the same time assist patients to master some basic essentials of rehabilitation training and psychological relaxation method; (6) Improve patient communication, understand the cause of psychological dynamic and mood swings, encourage family members to visit, make them enjoy affection fully and eliminate harmful stimulus. The patients' families should given short-term psychological knowledge training and the related guidance of caring skills, so that patient and their family can actively cooperate to complete the treatment.

\subsection{Evaluation was determined before treatment and 6 weeks after according to the evaluation methods.}

HAMD was evaluated by HAMD blindly, the raters didn't participate in treatment. According to the demarcation points set by Davis, JM [2], a score higher than 35 points refers to severe depression, higher than 20 points refers to mild or moderate depression, and lower than 8 points refers to no depression.

ADL assessment was made using the Modified Barthel Index of China rehabilitation research center [3]. The to- tal score was 100 points, more serious functional defect was between $0 \sim 20$, serious functional defect was between $25 \sim 45$, moderate functional defect was between 50 70, mild functional defect was between 75 95, state of caring oneself was 100 points.

\subsection{Statistical methods}

The data was process by SPSS 16.0 software package.

\section{Results}

There was no significant difference in HAMD and ADL scores of two groups before treatment, their depressive state was considerably improved after treatment, ADL was significantly increased, and patient on psychological care combined with Prozac was more effective than Prozac alone $(p<0.05)$. (See Table 1 and Table 2.)

\section{Discussion}

PSD was the common complication of stroke, foreign literature reported an incidence of $30 \% \sim 65 \%$. Since the patients unable care for themselves completely after stroke, it caused bad emotional, and severe patients may lose confidence in life, and even suicide. PSD patients usually did not cooperate with medical staff in early rehabilitation, and missed the best recovery time, which lead to a significant decrease of patient's quality of life and life satisfaction. Studies had shown that PSD was not only affected the recovery of neurological function, but may cause aggravated damages to the brain. Antidepressant drugs effectively treated depression, and prevented brain cell death and prompted the regeneration of cranial nerve associated with memory [4]. Therefore, appropriate and effective treatment given in time was necessary. At present, the pathogenesis of PSD remain unclear, however the occurrence of PSD was closely related to the physical and social psychological factors. The pathogenesis is generally considered as follows [5]: Primary endogenous theory stated that stroke cause

Table 1. HAMD scores of two groups before and after treatment.

\begin{tabular}{|c|c|c|c|c|c|}
\hline \multirow{2}{*}{ Group } & \multirow{2}{*}{ Case } & \multicolumn{2}{|c|}{$\mathrm{ADL}$} & \multirow{2}{*}{$t$ value } & \multirow{2}{*}{$p$ value } \\
\hline & & Before treatment & After treatment & & \\
\hline Experimental group & 28 & $28.34 \pm 3.55$ & $8.84 \pm 4.39$ & 8.25 & $<0.01$ \\
\hline Control group & 32 & $28.18 \pm 4.43$ & $13.41 \pm 5.82$ & 5.78 & $<0.01$ \\
\hline $\begin{array}{l}t \text { value } \\
p \text { value }\end{array}$ & \multicolumn{3}{|c|}{$\begin{aligned} & 5.59 \\
< & 0.05\end{aligned}$} & & \\
\hline
\end{tabular}

Table 2. ADL scores of two groups before and after treatment.

\begin{tabular}{|c|c|c|c|c|c|}
\hline \multirow{2}{*}{ Group } & \multirow{2}{*}{ Case } & \multicolumn{2}{|c|}{$\mathrm{ADL}$} & \multirow{2}{*}{$t$ value } & \multirow{2}{*}{$p$ value } \\
\hline & & Before treatment & After treatment & & \\
\hline Experimental group & 28 & $29.75 \pm 3.58$ & $60.24 \pm 8.39$ & 11.25 & $<0.01$ \\
\hline Control group & 32 & $30.52 \pm 3.81$ & $53.41 \pm 5.89$ & 13.78 & $<0.01$ \\
\hline $\begin{array}{l}t \text { value } \\
p \text { value }\end{array}$ & \multicolumn{3}{|c|}{$\begin{aligned} & 2.09 \\
< & 0.05\end{aligned}$} & & \\
\hline
\end{tabular}


damages in norepinephrine neurons and serotonin neurons and its pathway, leading the imbalance of norepinephrine and serotonin; and based on responsive mechanism theory, the loss of limbic function after stroke, transition of role, social function impairment, family relationships and other acute alteration were beyond the acceptable scope of patients, hence resulting physiological and psychological imbalance, so as to cause a pessimistic mood in patient and not willing communicate with people, eventually depression.

Psychological care helped patients in convalescence to know themselves, and paid attention to themselves, hence the problem able solved with the mature psychological defense mechanism. Besides that, it also corrected the wrong perception, establish a reasonable perception to treat disease accordingly, establish good social support system, at the same time, fully arouse the enthusiasm of family members, and relieve patients' psychological depression.

The novel antidepressant Prozac can selectively inhibit the reuptake of serotonin on presynaptic membrane, hence increase the concentration of serotonin in the synaptic cleft, and improve the function of serotonin in the brain consequently, which playing a good antidepressant effect. Therefore, the psychological care combined with Prozac targeted biology, society, psychology and other aspects to reduce patient's depressive symptoms.

The results showed a decreased HAMD and increased ADL in both groups after treatment. The HAMD, and ADL have statistical significance scores between two groups ( $p$ $<0.05)$. Suggesting that depression of patients was obviously improved, the recovery of neurological function was promoted and the quality of life of patients was enhanced. Psychological care combined with Prozac are more effective in the treatment of PSD.

\section{References}

1. The Chinese Neuroscience Society. The key points of diagnosis of cerebrovascular disease. Chinese Journal of Neurology. 1996; (6):379.

2. Zhang ZJ. Behavioral medicine scale handbook. Chinese Journal of Behavior Medical Sciences. 2000(special issue);147:134-135.

3. Department of medical administration in Ministry of Health of the People's Republic of China. Chinese rehabilitation medicine diagnosis and treatment standard. Beijing. Huaxia Publishing House. 1999:65.

4. Fann JR, Uomoto JM, Katon WJ. Cognitive improvement with treatment of depression following mild traumatic brain injury. Prezgl Lek. 1999;56(2):152-156.

5. Xiao G, Li HG. The influence of early intervention on depression after stroke. Journal of Clinical Psychosomatic Diseases. 2005;11(4):35l-352. 